\title{
Subacute progressive disseminated histoplasmosis in immunocompetent patient
}

\author{
Seong Jin Choi ${ }^{1,2}$, Han-Sol Choi ${ }^{1,2}$, June Young Chun ${ }^{1,2}$, Chung-Jong Kim ${ }^{1,2}$, Myung Jin Lee ${ }^{1}$, \\ Moonsuk $\mathrm{Kim}^{2}$, and Ghee Young $\mathrm{Choe}^{3}$
}

${ }^{1}$ Department of Internal Medicine, Seoul National University College of Medicine, Seoul; Departments of ${ }^{2}$ Internal Medicine and ${ }^{3}$ Pathology, Seoul National University Bundang Hospital, Seongnam, Korea

Received: March 13, 2015

Revised : April 20, 2015

Accepted: April 20, 2015

\section{Correspondence to}

Chung-Jong Kim, M.D.

Department of Internal Medicine, Seoul National University Bundang Hospital, 82 Gumi-ro 173beon-gil, Bundang-gu, Seongnam 13620, Korea

Tel: +82-31-787-7039

Fax: +82-31-787-7073

E-mail: erinusoo@gmail.com
To the Editor, Histoplasmosis is an endemic disease in Central and South America, and is caused by Histoplasma capsulatum [1]. It remains a frequent cause of opportunistic infection in patients whose immune systems are impaired by drugs or human immunodeficiency virus [2]. Clinical manifestations of histoplasmosis vary from an asymptomatic infection to a progressive disseminated histoplasmosis (PDH) involving the lung, gastrointestinal tract, endovascular structures, central nervous system, adrenal glands, and bone marrow [1].

Histoplasmosis also occurs in non-endemic areas, but at a much lower frequency. The most common cause is prior exposure in an endemic area [1]. Because of its nonspecific signs and symptoms, physicians in non-endemic areas should be able to recognize the conditions associated with histoplasmosis exposure to facilitate diagnosis and treatment.

South Korea is a non-endemic area and incidence of histoplasmosis has not been well characterized. Yoon et al. [3] reported that the prevalence of histoplasmosis was 0.054 per 100,000 persons in South Korea; however, that study had some limitations. The accuracy of their diagnosis could not be guaranteed because they only used secondary data collected from the Health Insurance Review and Assessment
Service database, and they did not distinguish between localized infections and disseminated infections. Furthermore, only a few case reports have been published, and most of these occurred in immunocompromised patients or patients with a non-systemic illness. Here, we report a case of disseminated histoplasmosis diagnosed in an immunocompetent adult who had recently traveled to the República de Honduras in Central America.

A 58-year-old male was seen in an outpatient clinic for a fever, generalized weakness, and weight loss of $10 \mathrm{~kg}$ over the previous month. The patient had previously done missionary work and been living in the República de Honduras beginning in March 2013. In September 2013, the following symptoms appeared: abdominal distension, fever, night sweating, and the development of a palpable mass on the neck. The patient visited a hospital in South Korea and a computed tomography (CT) of the neck with contrast and a colonoscopy were performed. The studies showed multiple necrotic lymphadenopathies in the neck and an ulcer in the ileocecal region. Pathologic findings of the ileocecal ulcer revealed granulomas in the submucosal area. At that time, tuberculous colitis or Crohn's disease was suspected, but the symptoms spontaneously resolved and the patient did not visit 

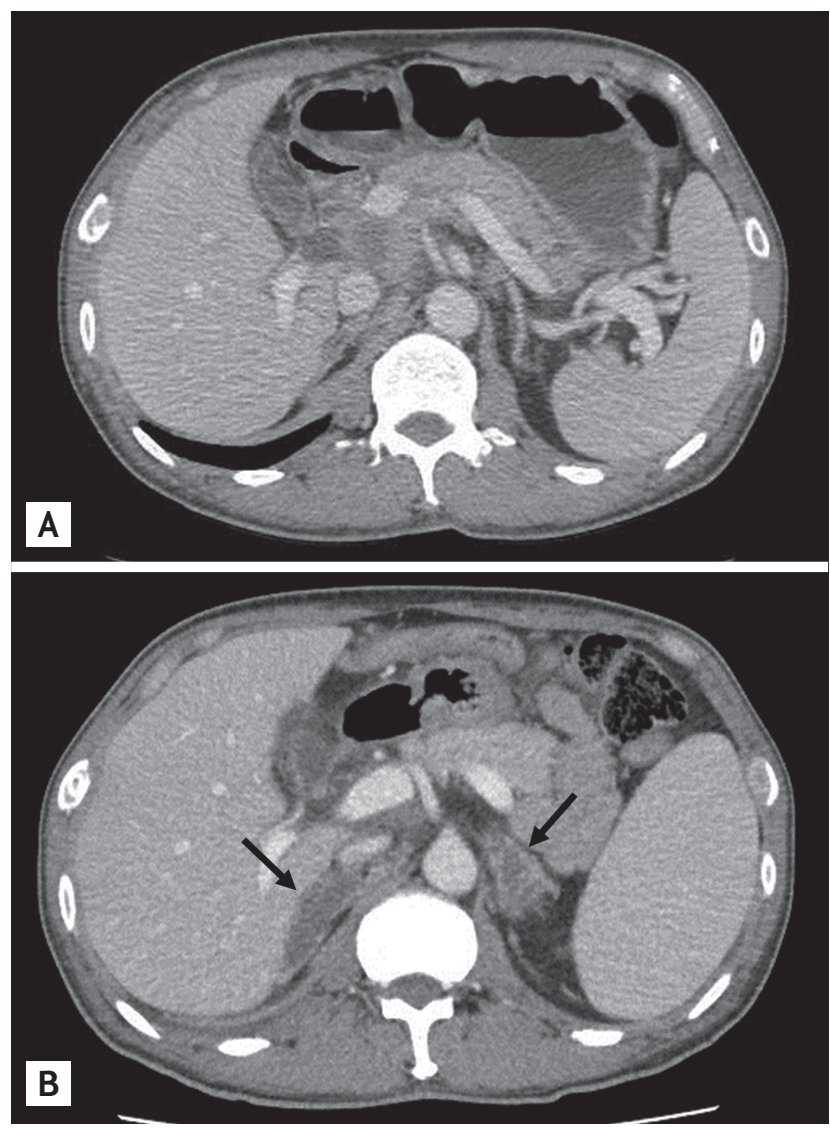

Figure 1. (A) Abdomen and pelvis computed tomography (CT) performed in September 2013 showed no abnormalities on both adrenal glands. (B) However, after 11 months, an abdomen and pelvis CT showed low-attenuated thickening of both adrenal glands (arrows).

the hospital again. In addition, an acid-fast bacillus stain and culture did not reveal Mycobacterium tuberculosis, and the patient returned to Honduras in December 2013.

The patient had been well until September 2014, at which point a fever, chills, generalized weakness, and a weight loss of $10 \mathrm{~kg}$ developed. The patient came to an outpatient clinic at our hospital and was admitted for further evaluation. Upon examination, the patient had a temperature of $38.3^{\circ} \mathrm{C}$, blood pressure of $122 / 66 \mathrm{mmHg}$, a pulse of 114 beats per minute, and a respiratory rate of 20 breaths per minute. A physical examination determined that the patient had no palpable lymph nodes on the neck, and the spleen was palpable by two finger points.

Laboratory findings revealed a hemoglobin level of 11.2 $\mathrm{g} / \mathrm{dL}$, a platelet count of $135,000 / \mu \mathrm{L}$, and a white blood cell (WBC) count of 3,540/ $\mathrm{L}$, containing $69 \%$ segmented neutrophils. Liver function tests determined the follow-
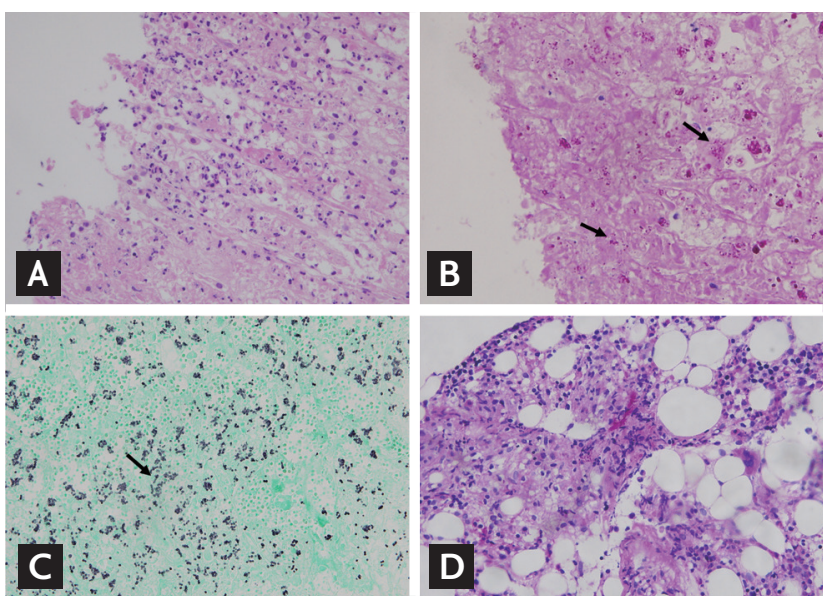

Figure 2. Adrenal gland biopsies (A, H\&E, ×400; B, Periodic acid-Schiff [PAS] stain, $\times 400$; C, Giemsa stain, $\times 400)$. (D) Bone marrow biopsy (PAS stain, $\times 400)$. The small yeast cells are visible by PAS staining (arrows).

ing results, aspartate aminotransferase $58 \mathrm{IU} / \mathrm{L}$, alanine aminotransferase $47 \mathrm{IU} / \mathrm{L}$, total bilirubin $0.7 \mathrm{mg} / \mathrm{dL}$, and albumin $3.0 \mathrm{~g} / \mathrm{dL}$. Serum calcium levels were $8.4 \mathrm{mg} / \mathrm{dL}$, lactate dehydrogenase levels were 294 IU/L, C-reactive protein levels were $4.15 \mathrm{mg} / \mathrm{dL}$ and serologic tests for hepatitis B, C, and human immunodeficiency virus were negative.

Chest radiography showed pneumonic infiltration on both lower lung fields. A chest CT revealed interlobular septal thickening and ground-glass opacity in both basal lung and abdomen, and a pelvis CT revealed bilateral low attenuated thickening of both adrenal glands, mild edematous wall thickening of the ascending colon and splenomegaly, which were not detected in the abdomen and pelvis CT performed in November 2013 (Fig. 1).

Hormone levels including plasma cortisol, serum adrenocorticotropic hormone, renin, aldosterone, urine metanephrine, and norepinephrine were within normal limits, which was indicative of normal adrenal gland function.

Gram staining and cultures from sputum and blood were negative. A bronchoscopy with bronchoalveolar lavage (BAL) was performed, and the BAL fluid was negative for an acid-fast stain and M. tuberculosis PCR. We obtained tissue from the right adrenal gland and ulceration in the terminal ileum by performing a percutaneous biopsy and colonoscopy, respectively. The adrenal gland showed extensive coagulation necrosis in the adrenal cortex with neutrophilic infiltration and numerous 
Periodic acid-Schiff (PAS) and Gomori methenamine sliver (GMS) positive fungal yeast, consistent with histoplasmosis. An ileal biopsy showed chronic active ileitis without granuloma, and PAS and GMS staining of the biopsy specimen from the colonoscopy also revealed numerous fungal yeast that were morphologically consistent with histoplasmosis (Fig. 2). The two specimens were also tested with an acid-fast stain and did not reveal the presence of M. tuberculosis. Histoplasma mycelial antibody and histoplasma yeast antibody complement fixation tests (Quest Diagnostics, Valencia, CA, USA) were positive with a titer of $\geq 1$ :32. A bone marrow exam and subsequent culture were performed due to leukopenia and thrombocytopenia aggravation that occurred on day 14 of hospitalization (WBC, 2,700/ $\mathrm{L}$; hemoglobin, $10.4 \mathrm{~g} /$ $\mathrm{dL}$; platelet count, $67,000 / \mu \mathrm{L}$ ), and the biopsy also showed fungal yeast and granulomas. The symptoms improved after administration of oral itraconazole $200 \mathrm{mg}$ twice daily and the patient was discharged without complications.

H. capsulatum is a soil-based fungus that has a dimorphic phase, the mycelial phase and the yeast phase. The former is present at ambient temperature and upon exposure to $37^{\circ} \mathrm{C}$, it changes to yeast cells. This transition is a critical determinant in the establishment of infection [2]. Inhalation of infectious particles from the soil is a major route of transmission leading to infection and human-to-human transmission has not been reported. Infected individuals harbor yeast, many of which can remain viable for many years. The finding that individuals who have moved from endemic to non-endemic areas may exhibit a reactivated infection after many years supports this long-term viability, although the mechanism of reactivation remains unknown [1].

Exposure to $H$. capsulatum is common for a person living in an endemic area, although the development of a symptomatic infection is rare [2]. Although the approximate epidemiology of histoplasmosis in Honduras has not been reported, surveys conducted in Latin American countries near Honduras have estimated the incidence of histoplasmosis to range from 0.1 to 0.29 cases per 100,000 persons [4].

In immunocompetent patients, histoplasmosis typically manifests as a self-limited respiratory infection. However, in immunocompromised hosts, disseminated histoplasmosis progresses aggressively within a few days and has a fatality rate of $100 \%$ if not treated. In adults who are not overtly immunosuppressed, exposure to soil containing infectious particles can be an important risk factor for contracting disseminated histoplasmosis, which progresses more slowly than in immunocompromised patients. However, if histoplasmosis is not treated, fatality rates of subacute or chronic PDH are similar to the acute form [5].

South Korea is a non-endemic country and physicians outside of endemic areas have less experience dealing with histoplasmosis. In addition, the clinical manifestations and histopathologic findings of histoplasmosis mimic those of tuberculosis. For this reason, PDH can easily be misdiagnosed as tuberculosis, with which physicians in South Korea are familiar. Other endemic mycoses including coccidioidomycosis, blastomycosis, and paracoccidioidomycosis are also important differential diagnoses when a patient has a history of travel to North or South America [3].

In this case, while this patient was not overtly immunosuppressed, he participated in the clearing of land during his travels, making this the potential source of infection. Initially, the acute hematogenous dissemination of histoplasmosis occurred during the first few weeks after exposure and with the development of cellular immunity, dissemination might have resolved without therapy. However, the patient returned to Honduras and was continuously exposed to H. capsulatum. After a second visit to another hospital, the patient was diagnosed with subacute $\mathrm{PDH}$ involving adrenal glands, colon, and the bone marrow. While the BAL fluid did not reveal histopathologic findings consistent with histoplasmosis, it is reasonable to assume that the pneumonic infiltration observed in both lungs was also the manifestation of histoplasmosis because inhalation is a major route of transmission.

Antifungal medications are commonly used to treat $\mathrm{PDH}$. Amphotericin B is recommended for patients who are sufficiently ill to require hospitalization. However, if the patient manifests with mild to moderate symptoms, itraconazole for 6 to 18 months is the preferred treatment over amphotericin B, which is inconvenient and generally not well-tolerated [5].

As travel increases, the number of individuals with a prior exposure to histoplasmosis in endemic areas, either through travel or residency, is expected to increase. Most 
immunocompetent people develop a benign, self-limited course of histoplasmosis. However, some patients can progress to subacute or chronic PDH if exposed to a large inoculum [1]. Therefore, we suggest that physicians outside of endemic areas should consider histoplasmosis in the differential diagnosis of patients with a history of travel to endemic areas, even if the immune system of the patient is not impaired.

Keywords: Histoplasmosis; Republic of Korea; Immunocompetence

\section{Conflict of interest}

No potential conflict of interest relevant to this article was reported.

\section{Acknowledgments}

We would like to thank Professor Hong-Bin Kim, Kyoung-Ho Song, Department of Internal Medicine, and Professor Jeong Su Park, Department of Laboratory Med- icine at Seoul National University Bundang Hospital, for assisting in the diagnosis of histoplasmosis.

\section{REFERENCES}

1. Wheat LJ. Histoplasmosis: a review for clinicians from non-endemic areas. Mycoses 2006;49:274-282.

2. Kauffman CA. Histoplasmosis: a clinical and laboratory update. Clin Microbiol Rev 2007;20:115-132.

3. Yoon HJ, Choi HY, Kim YK, Song YJ, Ki M. Prevalence of fungal infections using National Health Insurance data from 2009-2013, South Korea. Epidemiol Health 2014;36:e2014017.

4. Gomez BL. Histoplasmosis: epidemiology in Latin America. Curr Fungal Infect Rep 2011;5:199-205.

5. Wheat J, Sarosi G, McKinsey D, et al. Practice guidelines for the management of patients with histoplasmosis: Infectious Diseases Society of America. Clin Infect Dis 2000;30:688-695. 